\title{
Alguns Desafios Conceituais e Técnico- operacionais para o Desenvolvimento do Progra- ma de Saúde da Família como uma Proposta Transformadora do Modelo Assistencial
}

\author{
CESAR AUGUSTO ORAZEM FAVORETO ${ }^{1}$ \\ KENNETH ROCHEL DE CAMARGO JR.
}

Este artigo discute algumas questōes teórico-conceituais e técnico-operacionais que vêm fundamentando os serviços e as práticas dos profissionais médicos do Programa de Saúde da Família (PSF) no Brasil. Foram utilizados dados de três fontes distintas: a) textos que configuraram o debate internacional sobre promoçāo de saúde e saúde da família; b) documentos e propostas do Ministério da Saúde e c) dados produzidos em estudos empíricos sobre a implantação do PSF em algumas localidades no Brasil. Procura-se situar, no novo arranjo organizacional proposto pelo programa, os limites e desafios postos ao desenvolvimento de saberes e práticas de saúde capazes de contribuir para a ampliação da resolutividade e da integralidade das intervençōes individuais e coletivas em saúde, no âmbito da atenção básica do SUS.

Palavras-chave: Saúde da família; promoção da saúde; biomedicina; prática médica. 


\section{A Expansão do PSF e Seus Impasses}

Desde 1994, o Ministério da Saúde do Brasil vem introduzindo o Programa de Saúde da Família (PSF), no âmbito do SUS. Entretanto, foi a partir de 1998, com a efetiva transferência dos recursos do Piso de Atenção Básica (PAB fixo e variável) para os fundos municipais de saúde, que os municípios aceleraram a implantação das equipes de saúde da família.

O repasse dos recursos traduziu para os municípios, principalmente os de pequeno e médio portes, a prioridade política que o Ministério da Saúde vinha anunciando para a organização da atenção básica e, em particular, para o PACS (Programa de Agentes Comunitários de Saúde) e o PSF.

Essa expansão seguiu uma tendência de cobertura em áreas menos assistidas, representadas por pequenos municípios, distantes dos aglomerados urbanos, e/ou para cidades de médio porte (até 100 mil habitantes). A tendência ocorreu não somente pela lógica natural de ampliar a cobertura em áreas desassistidas mas, principalmente, pelo que representou, para os municípios menores, o poder de indução financeiro utilizado pelo Governo federal para execução dessa política. Por outro lado, o Ministério da Saúde priorizou a implantação do PSF nos municípios com baixos indicadores sociais e de saúde, procurando articulá-lo mais intensivamente a outras ações e políticas sociais do governo ${ }^{3}$.

Já em 1999, o Ministério reconhecia ter ocorrido um processo de pulverização das equipes de saúde da família nos municípios que tinham aderido ao programa, principalmente naqueles de médio e grande portes. As equipes vinham sendo implantadas, sobretudo, em áreas mais carentes e/ou de grande "risco sanitário". Isso foi considerado, naquele momento, como um fato que anularia a capacidade do PSF para desencadear um reordenamento dos sistemas municipais de saúde, isto é, transformar-se em estratégia estruturante dos sistemas locais de saúde. Para corrigir tal rumo, o Ministério da Saúde reajustou, em novembro de 1999, os valores dos incentivos para introdução do programa e criou uma nova modalidade para o seu cálculo, remunerando de forma diferenciada os municípios que apresentassem maior cobertura populacional pelas equipes de saúde da família.

No entanto, ao final de 2000 , as medidas de incentivo financeiro ainda não tinham sido suficientes para a ampliação do Programa de Saúde da Família nas grandes cidades do país. Das 10.402 equipes implantadas, a grande maioria encontrava-se em municípios com menos de 100 mil habitantes (Brasil/MS/COSAC, 2000). Entre as justificativas para esse quadro, 
Alguns Desafios Conceituais e Técnico-operacionais para o Desenvolvimento...

estão os elevados investimentos em recursos financeiros, humanos e materiais necessários para introdução ou ampliação da cobertura do PSF. Por conseguinte, foi criado um círculo vicioso que tem dificultado que grandes cidades atinjam, de acordo com os critérios vigentes, maiores valores de incentivo, redução do duplo gasto e das dicotomias no processo de transição entre a rede do PSF e a rede tradicional preexistente.

A despeito disso, são inegáveis alguns avanços na expansão do PSF. Estudo realizado pelo Ministério da Saúde, em 1999 - Avaliação da Implantação e Funcionamento do Programa de Saúde da Família - PSF - evidencia várias indicações do aumento da cobertura assistencial e do incremento de algumas ações programáticas produzidas pela implantação do PSF até então, tais como: significativo aumento no acesso às consultas médicas de pré-natal (de $43,8 \%$ para $93,7 \%$ ); elevação na utilização do Cartão da Criança no acompanhamento do crescimento e desenvolvimento infantil (de 53,1\% para 98,3\%); maior captação de hipertensos (de 13,9\% para $96 \%$ ), com concomitante aumento no tratamento desses pacientes (de $34,8 \%$ para $98,5 \%)^{4}$.

Em relação às limitações e precariedades do programa, o mesmo trabaTho identificou, como problemas principais, as condições físicas das unidades de PSF e a carência de recursos materiais minimamente necessários (como equipamentos e medicamentos) para assistência às gestantes e aos doentes crônicos nas unidades de saúde da família.

A essas análises e avaliaçōes sobre o desenvolvimento do PSF, parece necessário agregar uma abordagem qualitativa desse processo. Esta, por sua vez, deve permitir perceber melhor a forma como vêm sendo concebidas e realizadas as práticas em saúde no programa, tendo em vista o objetivo de contribuir para a promoção de transformações e melhoria do sistema de atenção básica à saúde.

Neste sentido, este estudo examinou a trajetória de implantação do programa em algumas realidades assistenciais locais, entrevistando gestores, gerentes e médicos do PSF nos municípios de Teresina, Rio de Janeiro e Volta Redonda ${ }^{6}$. Para subsidiar o trabalho, foram também consultadas avaliações de experiências de implantação do PSF dos municípios de Natal, Vitória da Conquista e Contagem ${ }^{7}$. Buscou-se identificar aspectos organizacionais e relativos às práticas em saúde que delineiam avanços e/ ou dificuldades para a consolidação do PSF como uma estratégia institucional estruturante de um novo modelo assistencial para a atenção básica.

A observação das práticas em saúde no PSF procurou compreender o 
modo como elas vêm sendo concebidas e realizadas a partir do novo arranjo organizacional proposto e qual o papel do programa na constituição de novos saberes e práticas de saúde capazes de contribuir para a resolutividade e a integralidade das intervenções sanitárias no SUS.

\section{Os Desafios Conceituais a Serem Enfrentados na Organização dos Serviços e das Práticas no PSF}

Na trajetória de implantação do PSF nos municípios estudados, tem predominado uma estratégia de extensão de cobertura assistencial, o que vem determinando um formato técnico e operacional para as equipes de PSF e sistemas municipais e regionais, caracterizado pela introdução de ações focalizadoras. Essas características, de certa forma, podem tornar-se fator limitante à sua evolução como estratégia substitutiva do modelo assistencial da atenção básica. Portanto, o caminho percorrido pela implantação do PSF, apesar das intenções em contrário, ainda tem sido marcado pela introdução de uma prática de medicina simplificada e dirigida às regiões e grupos sociais em situação de exclusão social e sanitária.

Entre as implicações da expansão do PSF como um projeto de extensão de cobertura, encontram-se questões como a legitimação social e política do programa e a capacidade resolutiva da atenção básica, principalmente se consideradas as necessidades e especificidades da atenção à saúde voltada às populações de grandes centros urbanos.

Em relação à resolutividade dos serviços de saúde da família, parece ser preciso enfrentar problemas, como: despreparo e qualificação insuficiente dos médicos de família; falta de mecanismos de relação do PSF com outros serviços (decorrente, entre outras, das dificuldades de desenvolvimento de sistemas de referência e contra-referência); precariedades das redes ambulatoriais e hospitalares locais preexistentes; e inadequação de um formato rígido para a composição dos profissionais das equipes, tendo em vista a existência de especificidades nas demandas entre as clientelas das diferentes áreas adscritas.

Autores como Paim e Mendes, entre outros defensores do PSF como substituto do modelo médico hegemônico, têm indicado condições e rumos para o programa, procurando evitar algumas das dificuldades anteriormente levantadas.

Assim, preocupado com que o PSF não reproduza acriticamente uma atenção primitiva, dirigida tão somente a populações mais carentes e/ou de 
Alguns Desafios Conceituais e Técnico-operacionais para o Desenvolvimento...

maior risco sanitário, Paim (1999) propõe a ampliação dos cuidados e de seus resultados por parte das equipes, aumentando a legitimação social e a visibilidade política do projeto. Nesta direção, sugere que o programa utilize mais intensivamente instrumentos epidemiológicos e se integre com a rede de serviços.

Como direcionamento para o programa, Mendes (1996) propõe a ênfase na "Promoção da Saúde" como estratégia diferenciadora do PSF em relação ao modelo tradicional, dando prioridade à "intervenção social" pelos serviços de saúde. Entende que esse modo de agir representaria uma reforma do modelo assistencial, baseada em um novo paradigma, o da "produção social da saúde". Na proposta defendida por Mendes, o trabalho dos profissionais estaria referido, genericamente, à saúde e, não exclusivamente, à medicina.

As posições desses autores reforçam as perspectivas apontadas pelo Ministério da Saúde para o PSF, como um modelo assistencial em que o foco esteja dirigido para a implementação de ações sobre a coletividade e cuja estruturação e direcionamento das intervenções individuais e coletivas estejam fundamentados no saber epidemiológico.

Contudo, o direcionamento dos serviços e práticas no PSF para ações coletivas de prevenção e promoção da saúde pode dar margem a entendimentos abstratos em relação à representação e à demanda da população. A clientela adscrita ao programa, ao procurar as unidades de saúde da família, traz demandas semelhantes às que trazia (e geralmente não era atendida) para as unidades tradicionais de saúde. Do mesmo modo que nestas, essa população busca, nas unidades do programa, respostas para necessidades singulares, como as expressas em suas angústias ou seus sintomas mal definidos. Tais problemas, entretanto, têm pouca relação com os tradicionais indicadores de morbimortalidade e não variam na mesma proporção, e do mesmo modo, caso ocorram mudanças significativas nos ambientes e nas relações sociais (Campos, 1997).

No processo de implantação e desenvolvimento do PSF, a perspectiva do cuidado aos indivíduos em suas demandas e formas singulares de adoecer e sofrer - e, conseqüentemente, na busca de atenção, por parte dos médicos ou enfermeiros de família - têm sido questōes ainda pouco aprofundadas, tanto pelos autores do campo da Saúde Coletiva como nos relatórios técnicos governamentais.

Os relatos de experiências locais de equipes de PSF de Teresina e Volta Redonda ${ }^{8}$ são ricos em descrever esses impasses e demonstram, claramente, as dificuldades que os profissionais vêm tendo para lidar e desenvolver 
Cesar Augusto Orazem Favoreto e Kenneth Rochel de Camargo Jr.

mecanismos para enfrentá-los. Vale dizer: em responder às demandas da população a que atendem.

A posição dos sanitaristas e de gestores em relação ao PSF como mudança do modelo assistencial enfatiza, principalmente, as formas de organização e controle, assim como a regulação e normatização dos processos de trabalho. Nessas abordagens, parece estar subentendido que as mudanças na dinâmica do trabalho das equipes e os novos produtos que poderão ser alcançados surgirão a partir da simples exposição dos profissionais de saúde a outros cenários de práticas e pela facilitação do acesso da população aos médicos e enfermeiras. Seriam essas condições, por si só, capazes de mudar o olhar, as atitudes e as habilidades dos profissionais frente à realidade de vida dessas pessoas e das comunidades que assistem?

Essas análises não atentam para outros aspectos presentes nas práticas em saúde, sobretudo em relação ao trabalho médico. Desconsideram que, por vezes, as mudanças nas condições e nos cenários das práticas não são, necessariamente, acompanhadas por um novo entendimento, por parte dos profissionais, de seus papéis e novos elementos em jogo em suas intervençōes.

Aqueles que planejam e gerenciam os serviços de saúde da família esperam que o profissional reproduza tecnicamente as práticas assistenciais, cientificamente comprovadas, legitimadas e quantificáveis pelo saber biomédico (como, por exemplo, o controle dos níveis pressóricos, a redução da glicemia etc.). Ao mesmo tempo, esperam e valorizam a geração espontânea de um comportamento mais "humano" ao lidar com os pacientes.

Possivelmente, resta para a maioria dos profissionais procurar, simplesmente, adaptar-se às novas condições de trabalho, muitas vezes de maneira mecânica e burocratizada, visto que o que dispõem como instrumento para compreender as novas situações em que estão inseridos é, apenas, o inquestionável - e limitado - saber técnico-científico estruturado na Biomedicina.

Nas situações em que esse saber não se mostra suficiente para lidar com questões como as relacionadas à vida, à subjetividade e às condições sociais, os profissionais de saúde tendem a se instrumentalizar de práticas que consideram "não-científicas" e que estariam mais próximas de suas qualidades individuais natas, como o carinho e a piedade, entre outras, adquiridas empiricamente durante suas vidas pessoais e profissionais.

O discurso que tem fundamentado a organização do PSF se baseia num tripé de idéias que consiste: (1) na crítica ao modelo médico flexneriano 
hegemônico; (2) na crítica às propostas de Medicina Comunitária (como uma medicina simplificada) e de Medicina de Família (como a reprodução da medicina liberal); e (3) na fundamentação teórico-conceitual da Saúde da Família, apresentada pelo discurso da "Promoção da Saúde".

Entretanto, esses pontos não parecem representar bases conceituais e técnico-operacionais suficientes para consolidar uma nova perspectiva para atenção à saúde pelo PSF, particularmente, se houver o objetivo de alcançar maior qualidade, resolutividade, humanização e integralidade nos cuidados prestados à população.

Os fatores limitantes para o desenvolvimento do PSF como modelo assistencial substituto vão além dos arranjos políticos, dos mecanismos financeiros indutores de mudanças ou dos instrumentos gerenciais de organização e avaliação dos serviços. O enfoque dado à estruturação do PSF precisa, também, considerar e aprofundar a discussão sobre os saberes e as crenças que podem estruturar as práticas da saúde da família, caso haja intenção de desenvolver um novo campo de transformações na atenção à saúde. Este novo campo de práticas se baseia em novos enfoques da relação entre as intervenções individuais e coletivas, assim como entre os saberes clínicos e epidemiológicos no cotidiano das ações dos profissionais de saúde.

\section{O Desafio de Promover Intervenções Coletivas e Sociais no Âmbito do PSF}

Nas entrevistas realizadas com os gerentes e/ou gestores do PSF de Volta Redonda e Teresina, foi identificada uma fala comum a todos: a citação da noção da "Promoção da Saúde" como orientadora dos serviços de saúde da família que estavam implantando.

Os gerentes manifestaram, a crença de que o PSF iria melhorar os indicadores sociais e de morbimortalidade dessas áreas tão carentes e relacionavam tal meta com a aplicação das ações de educação e promoção em saúde. Chamavam a responsabilidade para si e para o programa, admitindo que várias das carências a que as populações estão submetidas poderiam ser atenuadas com a presença do PSF. Neste sentido, valorizavam especialmente as equipes de saúde da família que, mais freqüente e diretamente, intervinham nas comunidades, através de ações de educação e atendimento domiciliar.

Habitualmente, contudo, as ações comunitárias pareciam depender de uma organização voluntariosa e pouco criteriosa por parte dos profissionais 
do PSF. Não foi identificada, na fala dos gerentes, uma preocupação mais profunda sobre o caráter técnico, pedagógico e ideológico que desse sustentação às intervenções no meio social e no ambiente.

As práticas mais freqüentemente referidas como de "Promoção da Saúde" eram as palestras para gestantes e mães vinculadas a programas de suplementação alimentar; palestras de sala de espera para portadores de doenças crônicas, voltadas para a redução de comportamentos de risco e adesão aos programas; a busca e atendimento domiciliar de pessoas faltosas aos programas ou sob risco à saúde, como gestantes fora do pré-natal e hipertensos ou diabéticos sem acompanhamento regular na unidade de saúde da família.

Já experiências com um caráter mais vivencial, como os grupos de convivência de pessoas idosas, eram, no geral, iniciativas decorrentes da participação de outras instituições fora da área da saúde.

Apesar de acreditar no potencial transformador das intervenções comunitárias por parte das equipes de saúde da família, os gerentes não deixavam de reconhecer que os problemas socioeconômicos e ambientais criavam grandes e profundas limitações às ações e interferiam na qualidade de vida. Responsabilizavam problemas estruturais - como o desemprego, a violência relacionada ao crime organizado, a fome, a precariedade das habitações, entre outras condições relacionadas à pobreza, à desigualdade social e ao modelo de desenvolvimento social, econômico e urbanístico - pelas dificuldades de vida e problemas de saúde das populações.

A despeito dos desgastes naturais decorrentes do amplo espectro de atividades em que estavam envolvidos no PSF, muitos profissionais se percebiam socialmente valorizados e demonstravam relevante noção de responsabilização com os problemas sociais e o vínculo estabelecido com a comunidade.

Da mesma forma, percebeu-se um alto grau de preocupação dos profissionais com o atendimento da demanda na unidade de saúde da família, com a eficácia do sistema de referência, como também com a distribuição de suas atividades dentro e fora das unidades.

As ações fora dos consultórios, isto é, voltadas à prevenção e promoção da saúde, eram mais valorizadas pelas enfermeiras do que pelos médicos das equipes. Entretanto, no geral, ambos compreendiam que estava, sob suas responsabilidades, a mudança dos hábitos e dos comportamentos considerados de risco à saúde por outros mais saudáveis, especialmente no grupo materno-infantil. 
Alguns Desafios Conceituais e Técnico-operacionais para o Desenvolvimento...

Em contato direto com a realidade de vida da população, os posicionamentos dos médicos e enfermeiras frente aos problemas sociais variavam bastante. Alguns experimentavam uma sensação de impotência; outros admitiam posturas caridosas em relação às situações que presenciavam, enquanto havia também aqueles que tinham uma atitude de reprovação quanto aos "maus hábitos", à "falta de educação" e de compreensão das pessoas nas comunidades.

As equipes dispunham de pouco apoio material, institucional ou mesmo de uma política social dos governos locais para o enfrentamento dos problemas sociais e ambientais das comunidades assistidas e para a implementação de ações de promoção da saúde. Nas ações de educação em saúde promovidas pelos médicos e enfermeiros eram valorizados os mecanismos de transmissão de informação, em especial o "ensino" de hábitos "saudáveis" de vida e de normas higiênicas. Com isso, muitos consideram que estão cumprindo seu papel na redução dos agravos à saúde.

A compreensão e o instrumental que os profissionais utilizam nas ações comunitárias estão circunscritos a uma concepção mecanicista e biologicista do processo saúde-doença. Este fato os leva a tomar atitudes que correm o risco de serem normatizadoras e moralizadoras dos comportamentos sociais e individuais. Os médicos expressaram, por vezes, surpresa com o alcance de algumas atividades desenvolvidas por organizações não-médicas ou, até mesmo, pelas iniciativas dos agentes comunitários de saúde junto à população. Essas ações pareciam ser mais efetivas na abordagem de temas relacionados à saúde e à qualidade de vida do que as atividades tradicionais que realizavam.

Observou-se, portanto, que os profissionais das unidades de saúde da família vivenciam, com freqüência, situações e dilemas nas suas relações com os aspectos socioculturais das comunidades e com as açōes de prevenção e promoção da saúde, tais como:

- a maioria das equipes está inserida em regiões submetidas a grandes dificuldades socioeconômicas e, apesar da crença imputada a esses profissionais no sentido de influírem na melhoria da qualidade de vida da população, eles percebem as dificuldades de mudar a realidade com os recursos disponíveis e a necessidade de políticas públicas que transcenderiam a área da saúde;

- o desejo de intervir sobre as condições de vida das comunidades adscritas não foi acompanhado pelo desenvolvimento e/ou reflexão das técnicas e habilidades de intervenção na dimensão coletiva das 
práticas em saúde. As ações de promoção da saúde ainda se organizam segundo um referencial normativo, biomédico e não-dialógico;

- a concepção dos gerentes e dos profissionais de saúde, assim como a própria organização do processo de trabalho das equipes, mantém rígidas divisões entre as intervenções de âmbito individual e coletivo e entre ações de prevenção, promoção e curativas.

Outro aspecto importante na análise das intervenções socioculturais é o risco de elas representarem movimentos de uniformização e enquadramento moral dos comportamentos individuais e coletivos exibidos pelas comunidades e, por conseguinte, contribuir para uma maior medicalização e controle social das comunidades assistidas pelo programa".

Ao considerar a importância das práticas sanitárias como elementos na construção da cidadania, da autonomia individual e coletiva, é preciso relativizar o papel das intervenções em saúde como forças propulsoras do dinamismo social, evitando os riscos antes citados. Isto não quer dizer que as práticas em saúde devam estar restritas, simplesmente, a uma dimensão técnica e médica. Ao contrário, as dimensões social, psicológica e cultural devem estar presentes não como externalidades, mas como um conjunto de saberes expressos em habilidades capazes de contextualizar as situações de sofrimento, seja do indivíduo ou de seu grupo social.

\section{O Desafio de Estruturar uma Prática de Saúde Dimensionada no Cuidado Integral aos Indivíduos}

A perspectiva de integrar dimensões de ordens distintas relacionadas à saúde humana torna-se intrinsecamente limitada, se os instrumentais utilizados nesse nível de intervenção permanecem restritos a um saber analítico, mecanicista e universalizante, como se caracteriza o modelo biomédico (Camargo, 1997; Rodrigues, 1980). Nesse modelo, persiste a dicotomia entre problemas da mente e do corpo, concebidos sob uma determinada racionalidade científica que, por si só, restringe uma compreensão mais holística e complexa do que significa o sofrer do homem, limitando-se à observação de lesões estruturais ou funcionais em um corpo físico ou no comportamento social.

À medida que as equipes do PSF passassem a conhecer e a participar do cotidiano da vida das pessoas em suas comunidades - inteirando-se de seus problemas e de suas representações sociais de saúde, doença e cura -, elas poderiam buscar construir uma abordagem que visualizasse o indiví- 
duo como integrante de uma sociedade que pensa, que tem sua história e crenças (Silva, 1996).

A expansão do PSF, em cenários como os grandes centros urbanos, obriga ao aprofundamento da análise do papel da Clínica na atenção prestada pelas equipes e, principalmente, pelos médicos do programa. As propostas envolvendo a atenção individual a ser prestada pelos serviços de saúde da família não devem persistir negando o papel da Clínica na organização das práticas, nem contornar esta discussão, enfocando e valorizando os mecanismos que, meramente, cumprem o papel de normatização e regulação dos atos médicos individuais.

Portanto, torna-se importante enfrentar o parco interesse e a dificuldade em especificar e abrir espaços para transformações na prática médica, questionando e/ou vislumbrando alternativas à Clínica, como esta vem sendo estruturada pelo modelo técnico-científico da Biomedicina.

São constatadas com frequiência as limitações ou as incongruências do modelo biomédico, mas as alternativas a ele, em geral, limitam-se a propostas que visam a reordenar, normatizar e avaliar os atos médicos, usando a epidemiologia e/ou o planejamento para tal fim. Contraditoriamente, os meios para operar as mudanças têm como referência (ou representam) disciplinas e saberes originários no próprio conhecimento biomédico ou são integrantes da mesma racionalidade técnico-científica que fundamenta a tão criticada Clínica.

Assim, o planejamento, a organização e a avaliação da atenção à saúde persistem considerando uma prática clínica limitada apenas ao escopo do método anatomoclínico. Não são abertas perspectivas para perceber que a Clínica, apesar de seguir no contexto dos fundamentos da Biomedicina (como foi bem definido nas análises do campo da Saúde Coletiva), tem seguido "teimando em ser mais um monte de outras coisas" (Campos, 1994).

É neste sentido que Campos (1994) alerta para o fato de que os esquemas que vêm sendo utilizados para explicar a prática médica e a Clínica têm-se limitado a compreensões teóricas e fechadas, sem perceberem possíveis descontinuidades e contradiçōes entre o modelo científico biomédico e as práticas clínicas.

Assim, parece importante focalizar a Clínica de maneira mais próxima do cotidiano de suas práticas, saberes e habilidades técnicas, no momento em que a atual reforma da atenção básica propõe o PSF como eixo estruturante da reorganização do modelo assistencial no Brasil.

Provavelmente, não bastam para a construção de um novo olhar clínico, como não bastaram, no século XVIII, apenas conjunturas políticas e cultu- 
rais propícias. O desenvolvimento do novo saber e de novas práticas implica uma nova disposição para conhecer a realidade, assim como uma transformação da clínica condizente com as diversas dimensōes envolvidas no processo de adoecer, incluindo não somente o olhar anatomoclínico mas, também, as percepções sociais e culturais do processo saúde-doença.

O desenvolvimento de uma prática clínica que avance sobre as limitações implícitas à racionalidade biomédica e ao modelo médico-liberal hegemônico deverá compreender mudanças no próprio saber e na racionalidade que a fundamentam e direcionam. Entretanto, é preciso pensar essas transformações para uma atividade que tem as características de um ofício e, portanto, deve compreender as condições dos cenários de atuação, o contato com outros saberes no cotidiano, bem como os mecanismos de reprodução do conhecimento e de comunicação interprofissionais.

Esse caminho ainda é frágil em relação à presença de modelos e conceitos consistentes e capazes de dar suporte a uma nova forma de conceber as intervenções em saúde. Contudo, é possível aferir que, para essa Clínica ampliada se organizar, produzir novas sínteses e sistematizações, fazem-se necessários alguns movimentos que visem (1) à interação com outros atores sociais e outros saberes (não limitados àqueles das biociências clássicas) e (2) ao questionamento do modo como são observadas e interpretadas as situações de adoecimentos das pessoas e a organização dos processos de trabalho, de produção e distribuição dos serviços em saúde - isto é, mudanças na forma como vêm sendo organizados os serviços e produzidas as intervenções em saúde (Campos, 1994).

Por outro lado, a ampliação da dimensão da Clínica na direção de uma prática voltada ao cuidado integral de pessoas pressupõe a valorização da fala e da escuta dos sujeitos envolvidos no cuidado em saúde e a concepção de que o limiar entre a saúde e a doença é singular, ainda que seja influenciado por planos que transcendem o estritamente individual, como o cultural e o socioeconômico, conforme sinalizam Coelho e Almeida Filho (1999).

É na valorização da fala e da escuta e, consequientemente, da narrativa como meio e fim da.prática clínica, que podem ser repensadas e transformadas as técnicas instrumentalizadoras e formuladoras do ato e do saber clínico. Desenvolvem-se assim habilidades e atitudes dos profissionais de saúde, ao integrarem, às suas práticas, elementos como a escuta, capaz de contextualizar as "estórias" de vida das pessoas; o silêncio receptivo, capaz de traduzir a acolhida sem preconceitos com aqueles que buscam atenção; o toque, que não apenas escrutina o interior dos corpos mas que oferece 
Alguns Desafios Conceituais e Técnico-operacionais para o Desenvolvimento...

materialidade e humanidade à relação que se estabelece entre duas pessoas; as posturas corporais e um discurso que incorporem a ética através de uma relação mais equiidistante entre aquele que cuida e quem é cuidado.

Estes, entre outros elementos, não são, necessariamente, novos componentes na formulação de intervenções voltadas à promoção, prevenção e/ou cura, mas podem significar novas sínteses, visando a uma integração ética da dimensão subjetiva e social ao tecnobiológico, ao revisitar e reinvestir em aspectos intrínsecos da dimensão humana ao cuidado em saúde. Relacionar esses elementos à Clínica visa a provocar uma abertura de espaço à subjetividade e ao social na prática médica - não mais como um aspecto circunstancial, baseado apenas em habilidades naturais como "dom e aptidão" e, portanto, desvinculado das estruturas de produção e das características da sociedade (Schraiber, 1995).

Portanto, rompe com concepções do ato médico que naturalizam artificialmente a apropriação das dimensões social e subjetiva à Clínica e impedem que os médicos as operem de fato e, não apenas, discursivamente. Conseqüentemente, as dimensões não-biomédicas do adoecer passam a compor o projeto de ação da prática clínica e a contribuir para reestruturar a relação médico-paciente em uma relação mais simétrica.

Aponta-se, assim, para possíveis mudanças nas práticas dos médicos ao lidar com o adoecer. Mais especificamente, promover mudanças na Clínica para que a mesma deixe de ser apenas um processo de desvelar diagnósticos mas se constitua efetivamente em um conjunto de crenças, posturas, habilidades e atitudes dirigidas a reduzir o sofrimento, a dor, a doença e evitar a morte. Deste modo, aproxima a intervenção clínica do cuidado e as pessoas, delineando novos saberes e um novo agir em saúde.

\section{Velhos e Novos Desafios}

Apontar para as carências de novos conhecimentos e saberes ou para a falta de respostas às necessidades cotidianas dos profissionais do PSF, mais do que um exercício acadêmico, torna-se uma necessidade, em vista dos grandes dilemas e questionamentos que os atores vivenciam quando passam a integrar de modo tão estreito os cenários de vida de suas clientelas.

Os médicos do PSF, a despeito de todo o investimento institucional que vem sendo feito pelas instâncias de governo, promovem ações de saúde individuais e/ou coletivas ainda limitadas pela velha dicotomia entre técnica e arte, ou melhor, entre uma prática humanista, baseada em um "dom" 
(pessoal e natural) do médico, e um saber biomédico técnico-científico, infalível e impessoal.

Reconhecer as limitações atuais do PSF enquanto uma estratégia de reestruturação da atenção à saúde - principalmente quando se tem como objetivo uma prática clínica ampliada e integradora das dimensões biopsicossociais do processo de adoecimento - não significa um descrédito em relação a essa modalidade de atenção. Pelo contrário: o modelo pode trazer, em seu bojo, oportunidades ímpares para o desenvolvimento dessas mudanças no cuidado em saúde e, particularmente, na prática clínica.

Pretendeu-se enfatizar que as transformações nas práticas passam pela emergência e valorização de novos saberes, frutos da maior penetração das dimensões subjetivas e sociais no campo biomédico; por uma postura mais dialógica dos prestadores de serviços de saúde com sua clientela (seja dos médicos entre si, das metodologias educacionais, dos instrumentos e atitudes gerenciais) e por uma abertura conceptual e científica em relação ao modelo biológico cartesiano e mecanicista, adotado pela Biomedicina.

Portanto, a perspectiva de reestruturação do modelo assistencial precisa contemplar o desenvolvimento das práticas e suas concepções, não se limitando a ser uma estratégia político-organizacional da rede de serviços de saúde.

\section{Referências Bibliográficas}

BELO HORIZONTE (MG). Prefeitura Municipal de Belo Horizonte. $B H$ Vida: projeto de saúde da família da Prefeitura de Belo Horizonte. Belo Horizonte, 2001. 112 p. (Mimeografado).

BRASIL. Ministério da Saúde. Coordenação de Saúde da Comunidade. Carta Consulta à Comissão de Financiamentos Externos. Brasília: Ministério da Saúde, Coordenação de Saúde da Comunidade, 2000.

BRASIL. Ministério da Saúde. Avaliação da Implantação e Funcionamento do Programa de Saúde da Família. Brasília: Ministério da Saúde, Secretaria de Assistência à Saúde, 2000.

CAMARGO Jr., K. R. A Biomedicina. Physis: Revista Saúde Coletiva, Rio de Janeiro, v.7, n.1, p. 45-68, 1997.

CAMPOS, G. W. Considerações sobre a arte e a ciência da mudança: revolução das coisas e reforma das pessoas: o caso da saúde. In: CECÍLIO, L .C. O. (Org.). Inventando a Mudança na Saúde. São Paulo: HUCITEC, 1994. p. 29-88. 
CAMPOS, G. W. Reforma da Reforma: repensando a saúde. São Paulo: HUCITEC, 1997. 220 p.

COELHO, M. T. A. D.; ALMEIDA FILHO, N. Normal - patológico, saúde-doença: revisitando Canguilheim. Physis: Revista Saúde Coletiva, v. 9, n. 1, p.13-36, 1999.

CONTAGEM (MG). Secretaria Municipal de Saúde. Prefeitura Municipal de Contagem. Relatório do processo de implantação do Programa de Saúde da Família do município de Contagem. Contagem, 2000. 10 p. (Mimeografado).

CONTAGEM (MG). Secretaria Municipal de Saúde. Prefeitura Municipal de Contagem. $1^{a}$ Amostra de saúde da família: os impactos da mudança. Contagem, 2000. 40 p. (Mimeografado).

FAVORETO, C.A.O. O PSF no Brasil: do discurso e das práticas. Rio de Janeiro, 2002. 126f. Dissertação (Mestrado em Saúde Coletiva) - Instituto de Medicina Social, Universidade do Estado do Rio de Janeiro.

FOUCAULT, M. Vigiar e Punir: nascimento da prisão. Petrópolis: Vozes, $1977.280 \mathrm{p}$.

MENDES, E.V. Uma agenda para a saúde. São Paulo: HUCITEC, 1996. $300 \mathrm{p}$.

PAIM,J.S. A Reforma Sanitária e os Modelos Assistenciais. In: ROUQUAYROL, MZ; ALMEIDA FILHO, N. Epidemiologia e Saúde. 5. ed. Rio de Janeiro: MEDSI, 1999. p. 473-488.

PINHEIRO, R. Da defesa do aço à defesa da vida: o cotidiano dos atores em suas práticas nos serviços de saúde: o caso de Volta Redonda. Rio de Janeiro, 2000. 174 f. Tese (Doutorado em Saúde Coletiva) - Instituto de Medicina Social, Universidade do Estado do Rio de Janeiro.

ROCHA, N.S.P.D. Análise do Programa Saúde da Família no município de Natal-RN: inovações assistenciais? Rio de Janeiro, 2000. 197f. Dissertação (Mestrado em Saúde Coletiva) - Instituto de Medicina Social, Universidade do Estado do Rio de Janeiro.

RODRIGUES, R. D. A crise da medicina: prática e saber. Rio de Janeiro, 1980. 128f. Dissertação (Mestrado em Saúde Coletiva) - Instituto de Medicina Social, Universidade do Estado do Rio de Janeiro.

SCHRAIBER, L B. Políticas públicas e planejamento nas práticas de saúde. Saúde em Debate, n. 47, 1995.

SILVA, S. F. Programa de Saúde da Família: o médico de família. In: - A construção do SUS a partir do Município: etapas para a municipalização plena da saúde. São Paulo: HUCITEC, 1996. p. 83-87. 
SOUZA, S. P. S. A inserção dos médicos no serviço público de saúde: um olhar focalizado no Programa de Saúde da Família. Rio de Janeiro, 2001. 130 f. Dissertação (Mestrado em Saúde Coletiva) - Instituto de Medicina Social, Universidade do Estado do Rio de Janeiro. VITÓRIA DA CONQUISTA (BA). Prefeitura Municipal de Vitória da Conquista. Relatório sobre o Sistema Municipal de Saúde: Saúde - Vitória da Conquista. Vitória da Conquista, 2000. 43 p. (Mimeografado).

\section{NOTAS}

1 Médico, professor assistente do Departamento de Medicina Interna da Faculdade de Ciências Médicas da UERJ, mestre em Saúde Coletiva pelo IMS/UERJ.

2 Médico, professor adjunto do Departamento de Planejamento e Administração em Saúde do IMS/UERJ, Doutor em Saúde Coletiva pelo IMS/UERJ.

3 Como o Programa Comunidade Solidária e os Programas de Redução da Mortalidade na Infância, de Intensificação das Ações para Ređução da Mortalidade Materna e o Plano de Apoio aos Estados de Menor Desenvolvimento Humano - IDH 14 (Projeto Alvorada).

4 Outras melhorias mencionadas pelo mesmo estudo incluem: crescimento na coleta de exames preventivos de câncer cérvico-uterino (de $16,1 \%$ para $67,5 \%$ ); aumento no cadastramento de pacientes diabéticos (de $12,7 \%$ para $93,9 \%$ ), bem como seu tratamento (de $32,5 \%$ para $97 \%$ ); elevação na busca ativa de pacientes com tuberculose, de $14,8 \%$ para $87,3 \%$ (Brasil, 2000).

5 Este estudo foi realizado como parte do trabalho de campo da dissertaçāo de mestrado defendida em 2002 por um dos autores (Cesar Augusto O. Favoreto), no Instituto de Medicina Social da UERJ, intitulada $O$ PSF no Brasil: do discurso e das práticas, orientada pelo professor Kenneth Rochel de Camargo Jr.

6 Os critérios de elegibilidade dos municípios foram: ser municípios de grande porte populacional (mais de 100 mil habitantes), existirem uma rede de serviços tradicionais bem estabelecidos e as condições operacionais de viabilidade do estudo. Nāo houve intenção de realizar estudos de casos de implantaçāo do PSF, nem de produzir comparações ou fazer correlações entre as experiências. Pelo contrário, o interesse foi observar situaçōes diferentes, de modo que pudesse ser extraída maior diversidade de elementos técnico-gerenciais e das práticas em saúde das observações realizadas. Outro determinante é a diversidade quanto ao grau de desenvolvimento e representatividade do PSF em relação à estrutura assistencial.

7 Os trabalhos consultados foram: SMS Contagem (2000a; 2000b); Rocha (2000); Prefeitura Municipal de Vitória da Conquista (2000).

8 Igualmente são ricos os relatos dos médicos do PSF dos municípios de Camaragibe (PE) e Sobral (CE), colhidos e analisados por Souza (2001).

9 A perspectiva de controle social pela medicina foi desenvolvida com muita propriedade por Foucault (1986), em Vigiar e punir. 


\section{ABSTRACT}

Some conceptual and technical/operational challenges for the development of the Family Health Program as a proposal to change the health care model in Brazil

This article discusses key theoretical/conceptual and technical/operational issues underlying the services provided and practices performed by physicians in the Family Health Program (PSF) in Brazil. Three different data sources were used; a) literature from the international debate on health promotion and family health; b) documents and proposals by the Brazilian Ministry of Health; and c) data from empirical studies on the implementation of the PSF in selected areas of Brazil. Within the new organizational framework proposed by the Program, the article attempts to identify the limits and challenges to the development of health-related knowledge and practice capable of helping expand the case-resolving capacity and integrality of individual and collective health interventions within the basic care provided under the Unified Health System (SUS).

Keywords: Family health; health promotion; biomedicine; medical practice.

Recebido em: 29/05/02

Aprovado em: 10/06/02 